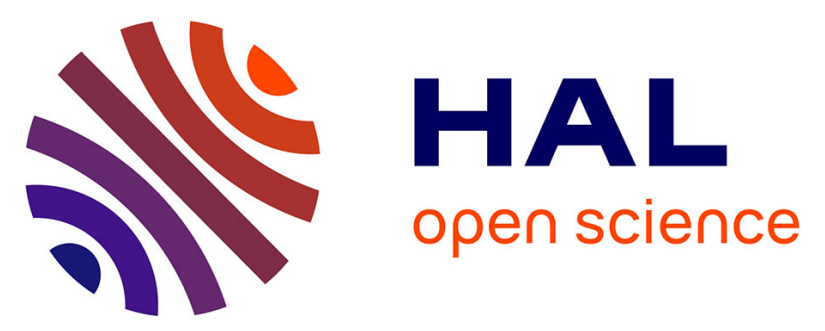

\title{
Apport des marqueurs isotopiques et biogéochimiques dans la reconstitution du paléoenvironnement de la grotte du Lazaret (Nice, Alpes-Maritimes) au cours du Pléistocène supérieur (stade isotopique 5)
}

Louis Rousseau, Jacques Beauchamp, Christophe Falguères, Christophe

Emblanch, Dominique Genty, Jean-Jacques Bahain, Dominique Blamart

\section{To cite this version:}

Louis Rousseau, Jacques Beauchamp, Christophe Falguères, Christophe Emblanch, Dominique Genty, et al.. Apport des marqueurs isotopiques et biogéochimiques dans la reconstitution du paléoenvironnement de la grotte du Lazaret (Nice, Alpes-Maritimes) au cours du Pléistocène supérieur (stade isotopique 5). Comptes Rendus Géoscience, 2005, 337 (15), pp.1348-1354. 10.1016/j.crte.2005.08.006 . hal-02295153

\section{HAL Id: hal-02295153}

https://hal-univ-avignon.archives-ouvertes.fr/hal-02295153

Submitted on 12 Oct 2020

HAL is a multi-disciplinary open access archive for the deposit and dissemination of scientific research documents, whether they are published or not. The documents may come from teaching and research institutions in France or abroad, or from public or private research centers.
L'archive ouverte pluridisciplinaire HAL, est destinée au dépôt et à la diffusion de documents scientifiques de niveau recherche, publiés ou non, émanant des établissements d'enseignement et de recherche français ou étrangers, des laboratoires publics ou privés. 
Géochimie (Géochimie isotopique)

\title{
Apport des marqueurs isotopiques et biogéochimiques dans la reconstitution du paléoenvironnement de la grotte du Lazaret (Nice, Alpes-Maritimes) au cours du Pléistocène supérieur (stade isotopique 5)
}

\author{
Louis Rousseau $^{\mathrm{a}, \mathrm{b}}$, Jacques Beauchamp ${ }^{\mathrm{b}, *}$, Christophe Falguères ${ }^{\mathrm{a}}$, \\ Christophe Emblanch ${ }^{\mathrm{c}}$, Dominique Genty ${ }^{\mathrm{d}}$, Jean-Jacques Bahain ${ }^{\mathrm{d}}$, Dominique Blamart ${ }^{\mathrm{e}}$ \\ a Département de préhistoire du Muséum national d'histoire naturelle, UMR 5198 du CNRS, 1, rue René-Panhard, 75013 Paris, France \\ ${ }^{\mathrm{b}}$ Laboratoire de sédimentologie et géochimie, faculté des sciences, 80039 Amiens cedex, France \\ ${ }^{c}$ Laboratoire d'hydrogéologie, université d'Avignon, 33, rue Louis-Pasteur, 84000 Avignon, France \\ d IPSL/Laboratoire des sciences du climat et de l'environnement, L'Orme des Merisiers, CEA Saclay, 91191 Gif-sur-Yvette cedex, France \\ e IPSL/Laboratoire des sciences du climat et de l'environnement, bât. 12, av. de la Terrasse, 91198 Gif-sur-Yvette, France
}

Reçu le 10 mars 2004 ; accepté après révision le 17 août 2005

Disponible sur Internet le 8 septembre 2005

Présenté par Georges Pédro

\section{Résumé}

Les variations de composition isotopique de la calcite du plancher stalagmitique E sont interprétées comme des indices de changement de circulation atmosphérique et de couverture végétale au cours du stade IOS 5. Les variations du $\delta^{18} \mathrm{O}$ seraient la conséquence du changement de la source des précipitations. Pendant un sous-stade chaud, les précipitations seraient d'origine méditerranéenne, alors qu'elles seraient d'origine atlantique pendant un sous-stade froid. Les variations $\mathrm{du} \delta^{13} \mathrm{C}$ traduiraient les variations de la couverture végétale et de la nature du sol. La végétation serait de type arboré, comme l'indiquent les spectres polliniques et comme le confirment les molécules organiques piégées dans la calcite : stérols, terpénoïdes, acides humiques. Pour citer cet article : L. Rousseau et al., C. R. Geoscience 337 (2005).

@ 2005 Académie des sciences. Publié par Elsevier SAS. Tous droits réservés.

\section{Abstract}

Isotopic and biogeochemical markers help in reconstructing the Lazaret Cave environment (Nice, France) during Upper Pleistocene (IOS 5). The isotopic composition of calcite from the stalagmitic floor $\mathrm{E}$ of the Lazaret Cave is interpreted as proxy of atmospheric circulation and vegetal cover changes during IOS 5. The $\delta^{18} \mathrm{O}$ variations could indicate change in precipitation sources, which could originate from the Mediterranean Sea during warm periods and from the Atlantic Ocean during colder periods. The $\delta^{13} \mathrm{C}$ variations could be related to vegetal cover and soil type. Tree cover appears to be dominant according to pollen spectra, and organic molecules trapped into calcite (sterols, terpenoids, humic acids). To cite this article: L. Rousseau et al., C. R. Geoscience 337 (2005).

○ 2005 Académie des sciences. Publié par Elsevier SAS. Tous droits réservés.

\footnotetext{
* Auteur correspondant.

Adresse e-mail : j.beauchamp@u-picardie.fr (J. Beauchamp).
} 


\section{Présentation générale}

La grotte du Lazaret est située en bordure de la mer Méditerranée, dans la ville de Nice elle-même (Fig. 1). Ce site, connu dès le début du XIX ${ }^{\mathrm{e}}$ siècle, a fait l'objet de fouilles méticuleuses [17,22], qui ont notamment fourni des restes humains, attribués à des anténéandertaliens [19], associés à une industrie de l'Acheuléen supérieur.

De Lumley [17] a divisé le remplissage archéologique en trois sous-unités (CI, CII et CIII, Fig. 2), précisées ensuite par de Lumley et Tavoso [18]. Les dépôts sont constitués principalement d'une succession de blocs et de débris de roches, piégés dans une matrice argilo-sableuse faiblement consolidée. Un plancher stalagmitique (E) étendu, dont l'épaisseur varie et peut atteindre une vingtaine de centimètres, recouvre, dans le fond de la grotte, les dépôts archéologiques du Pléistocène moyen (Fig. 1). Les datations radiométriques montrent que le début du concrétionnement du plancher s'est produit aux alentours de $-130 \mathrm{ka}$, alors que la fin de la cristallisation se situe vers -70 à -60 ka $[8,30]$.

Afin de reconstituer les fluctuations climatiques contemporaines de la formation du plancher, une analyse palynologique a été faite par Gauthier [11]. Dans les échantillons prélevés dans le plancher stalagmitique $\mathrm{E}$, les grains de pollen sont absents ou rares. Il n'est donc pas possible de déceler des variations nettes dans les spectres polliniques entre -100 et $-70 \mathrm{ka} \mathrm{BP}$, bien que des fluctuations climatiques globales soient bien connues dans cet espace de temps.

Il apparaît donc nécessaire d'utiliser d'autres marqueurs. Nous aurons recours à des critères géochimiques en analysant dans la calcite les isotopes de l'oxygène, les isotopes du carbone et des composés organiques, comme les stérols.

\section{Méthodes et résultats}

\subsection{Données radiométriques}

Deux tranchées ont été réalisées dans le plancher stalagmitique (E), ainsi que des carottages. Des analyses radiométriques ont été effectuées par l'un de nous sur 22 échantillons au Geological Survey's Menlo Park Laboratory. La teneur des différents isotopes a été évaluée par spectrométrie alpha. Les carottes K et L (Fig. 3a) présentent des dates similaires à celles obtenues sur les échantillons J et 89-16. De plus, des datations TIMS ont été réalisées par Shen et Turpin (communication écrite) sur des échantillons de la tranchée TRA (Figs. 2 et 3b); elles s'échelonnent entre $112 \mathrm{ka}$ à la base et $62 \mathrm{ka}$ au sommet. Ces valeurs confirment que la période de croissance du plancher stalagmitique s'est produite en grande partie au cours du stade OIS 5.

\subsection{Données isotopiques}

L'analyse isotopique a été menée sur les isotopes stables de l'oxygène $\left(\delta^{18} \mathrm{O}\right)$ et du carbone $\left(\delta^{13} \mathrm{C}\right)$, d'après le protocole de Shackleton [29]. Les résultats des analyses du $\delta^{13} \mathrm{C}$ et du $\delta^{18} \mathrm{O}$ du plancher stalagmitique $\mathrm{E}$ de la grotte de Lazaret sont présentés sur la Fig. 4. Les valeurs de $\delta^{13} \mathrm{C}$ et de $\delta^{18} \mathrm{O}$ sont exprimées en $\delta \%$ par rapport au PDB. Sur la même figure, nous avons porté, à des fins de comparaison, la variation de composition isotopique en oxygène de tests de foraminifères benthiques issus d'une carotte océanique analysée par Pisias et al. [25].

\subsubsection{Oxygène}

Sur la Fig. 4, la teneur en ${ }^{18} \mathrm{O}$ de la calcite varie, et ces variations se retrouvent dans les courbes marines. On reconnait les sous-stades définis en milieu marin. Le $\delta^{18} \mathrm{O}$ de la calcite varie entre $-3,9 \%$ pour un sousstade chaud (OIS 5.3) et $-5,7 \%$ pour un sous-stade froid (OIS 5.2). Cependant, les variations sont en partie de sens opposé : pendant deux périodes chaudes du stade isotopique 5 (OIS 5.1, 5.3), le $\delta^{18} \mathrm{O}$ diminue en milieu marin, alors qu'il crô̂t dans la calcite. En revanche, la corrélation est moins claire pour le stade 5.5, ce qui laisse supposer l'intervention d'autres facteurs. On peut citer l'équilibre isotopique, la température régnant lors de la précipitation de la calcite, le $\delta^{18} \mathrm{O}$ de l'eau d'infiltration, lui-même dépendant de la température extérieure, du chemin suivi par les dépressions et du $\delta^{18} \mathrm{O}$ de l'océan [24].

En milieu continental, le $\delta^{18} \mathrm{O}$ est contrôlé par la température et la composition de l'eau. En particulier, le signal isotopique des spéléothèmes dépend des conditions climatiques de la surface, du système de percolation et des conditions de précipitation de la calcite, qui se fait par dégazage et évaporation. Outre la tempéra- 


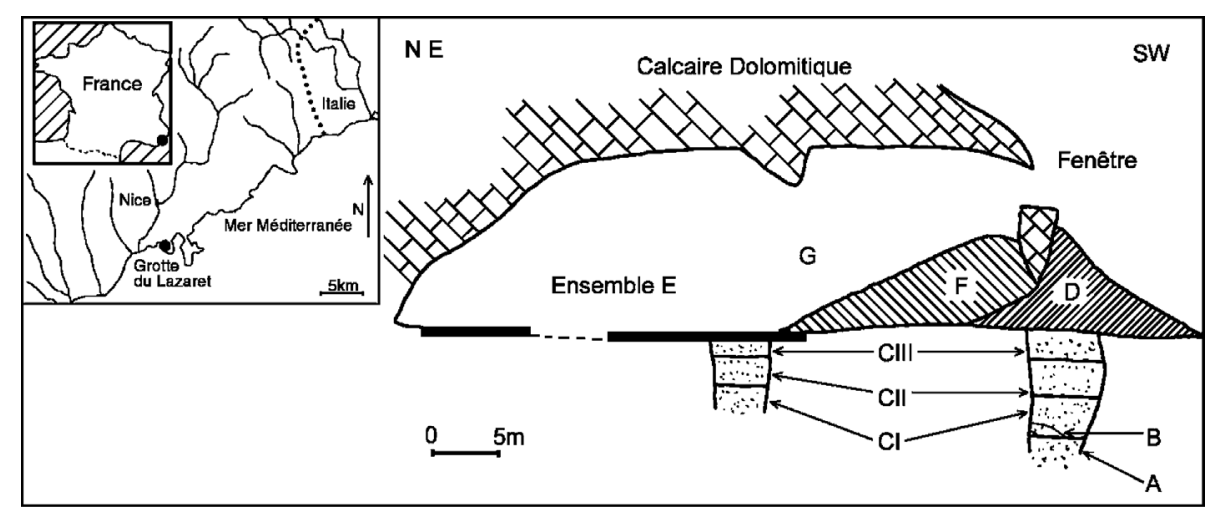

Fig. 1. Localisation et coupe dans la grotte du Lazaret (d'après [17], modifié) : A, B, niveaux marins; $\mathbf{C}$, remplissage : cailloutis et argiles; $\mathbf{D}, \mathbf{F}$, brèches de fermeture; $\mathbf{E}$, plancher stalagmitique.

Fig. 1. Location sketch and section in the Lazaret Cave (modified from de Lumley [17]): A, B, marine strata; C, pebbles and clay filling; D, F, collapse breccia; E, stalagmitic floor.

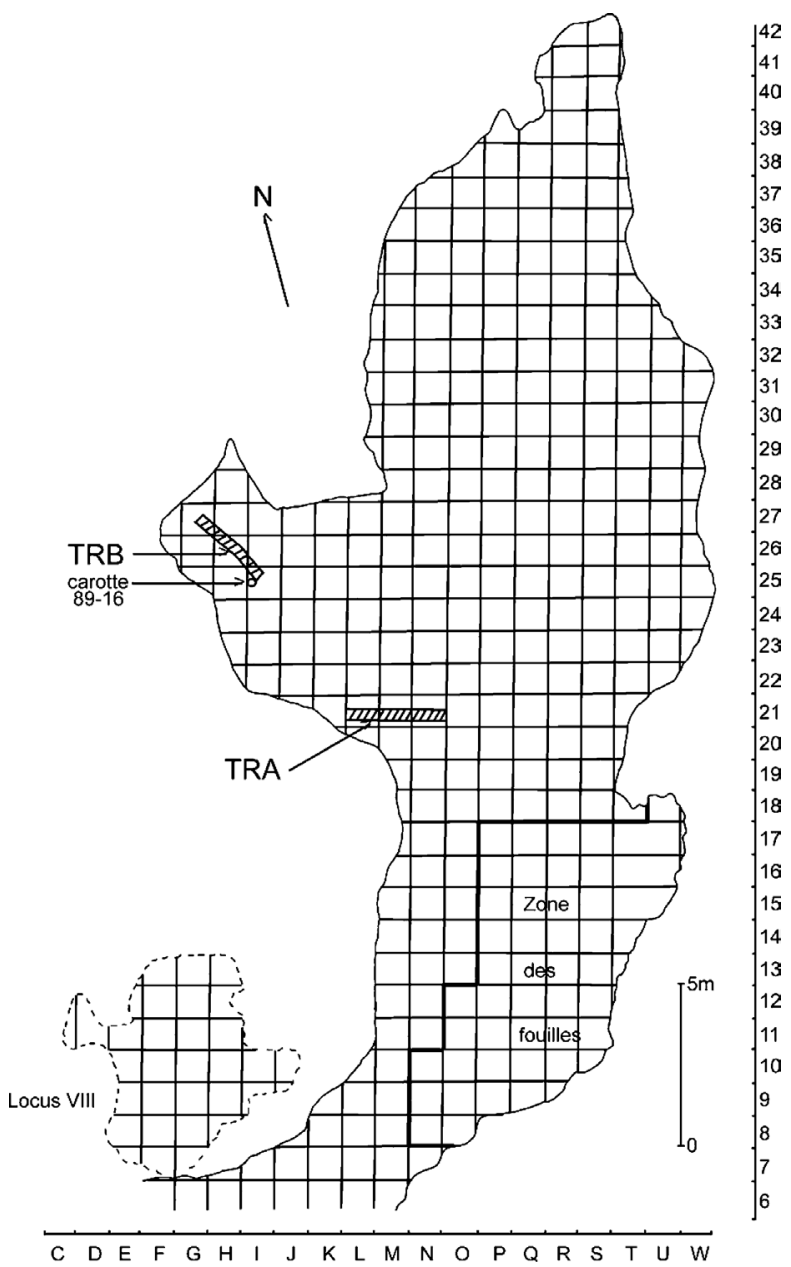

Fig. 2. Localisation de la carotte 89-16 et des tranchées TRA et TRB sur le plan de carroyage de la grotte du Lazaret.

Fig. 2. Position of core 89-16 and TRA and TRB ditches on the location grid of the Lazaret Cave. ture, l'abondance des précipitations joue également un rôle.

Selon les travaux antérieurs, les rapports existant entre le $\delta^{18} \mathrm{O}$ des spéléothèmes, le $\delta^{18} \mathrm{O}$ du milieu marin et la température sont loin d'être simples. Les travaux classiques de Winograd et al. [34] sur la veine de calcite de Devil's Hole (États-Unis) montrent des variations en opposition par rapport au $\delta^{18} \mathrm{O}$ marin. Quin Jamin [26] a montré, dans le karst de Guilin (Chine du Sud), que le $\delta^{18} \mathrm{O}$ des spéléothèmes était élevé en climat froid et sec, mais faible en climat chaud et humide. Johnson et Ingram [14] ont trouvé une corrélation inverse du $\delta^{18} \mathrm{O}$ avec la température dans les spéléothèmes anciens, mais une corrélation directe dans les concrétions actuelles.

L'influence prédominante des précipitations est soulignée par Bar-Matthews et al. [1] en région semi-aride : le $\delta^{18} \mathrm{O}$ est grand en saison sèche par forte évaporation. Frumkin et al. [10] arrivent à la même conclusion en climat méditerranéen. Sous climat tempéré océanique, Williams et al. [33] trouvent une corrélation positive avec la température; ils concluent à l'influence de la source océanique.

La grotte du Lazaret est située au bord de la mer : on peut s'attendre à retrouver l'influence de «l'effet de source». Actuellement, les vents dominants sont ceux d'est et de sud-est; ils apportent les pluies du golfe de Gènes, c'est-à-dire de la source méditerranéenne enrichie en ${ }^{18} \mathrm{O}\left(\delta^{18} \mathrm{O}\right.$ compris entre 0,5 et $\left.1,5 \%\right)$. Ce schéma de circulation atmosphérique doit pouvoir s'appliquer aux périodes plus chaudes de l'interglaciaire du stade isotopique 5 (sous-stades OIS 5.1, 5.3, 5.5). Pendant les période froides (sous-stades OIS 5.0, 5.2, 5.4), la circulation océanique et atmosphérique était modifiée sur l'Atlantique nord [4,15], les dépressions nordatlantiques passaient plus au sud de l'Europe; elles ap- 


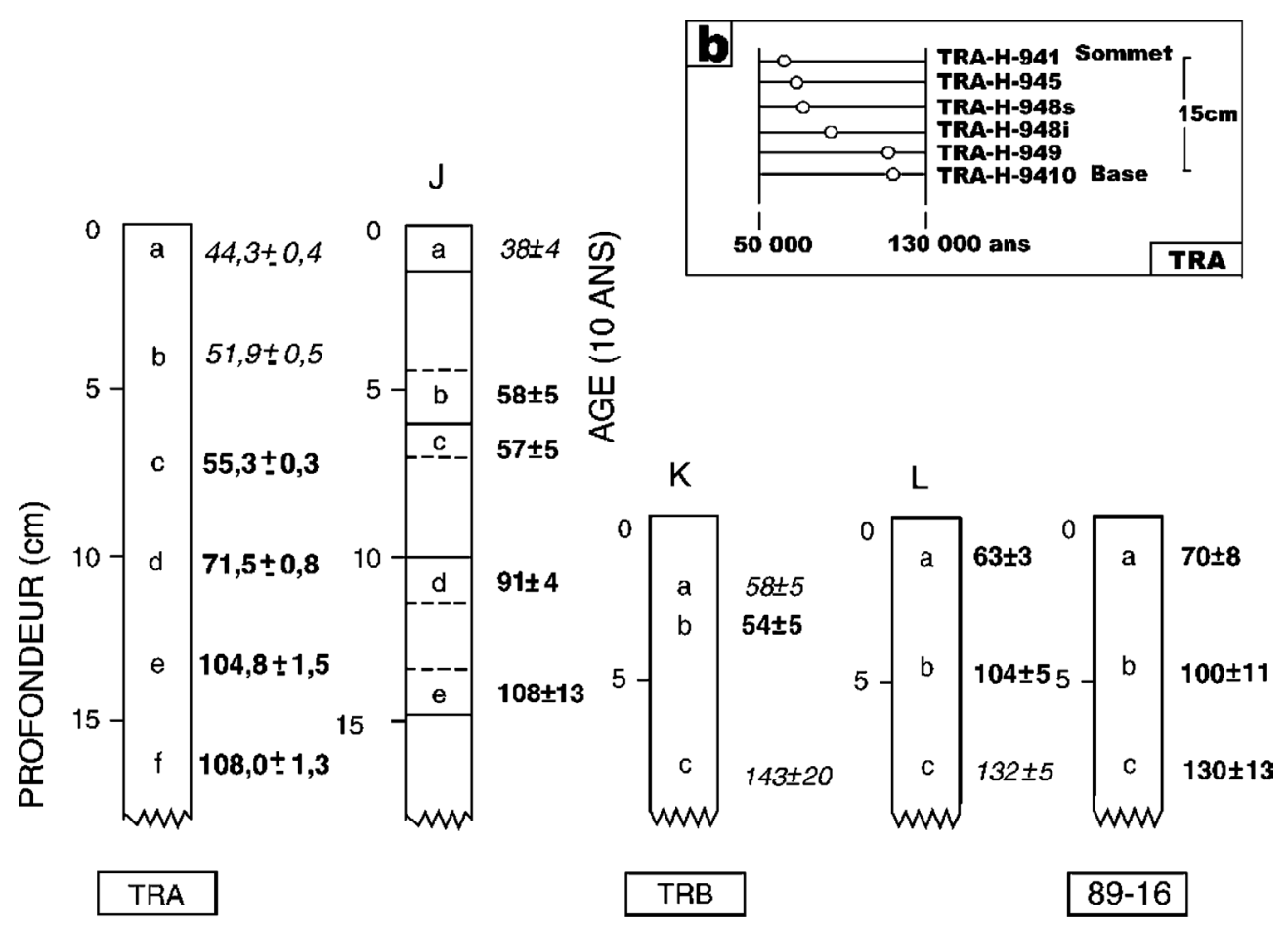

Fig. 3. Datation de la carotte et des tranchées (les chiffres à gauche indiquent l'épaisseur en centimètres) par spectrographie alpha (a) et TIMS (b). Les valeurs en italiques correspondent à des échantillons montrant une forte contamination (rapport ${ }^{230} \mathrm{Th} /{ }^{232} \mathrm{Th}$ faible) [8].

Fig. 3. U-series dating of core 89-16 and TRA and TRB ditches by alpha spectrography (a) and TIMS (b). Data in italic fonts are not reliable because of contaminated samples $\left({ }^{230} \mathrm{Th} /{ }^{232} \mathrm{Th}\right.$ low ratio) [8].

portaient des précipitations de source atlantique plus pauvres en ${ }^{18} \mathrm{O}\left(\delta^{18} \mathrm{O}\right.$ compris entre 0 et $1 \%$ ). Les fluctuations observées dans le $\delta^{18} \mathrm{O}$ de la calcite du plancher apparaissent corrélées positivement avec les variations de température, mais elles matérialiseraient en fait les fluctuations de la circulation atmosphérique.

\subsubsection{Carbone}

Sur la même Fig. 4, la teneur en $\delta^{13} \mathrm{C}$ de la calcite varie et ces variations accompagnent celles du $\delta^{18} \mathrm{O}$. L'amplitude maximale de la variation s'étend de $-9,8 \%$ en période chaude (OIS 5.5) à $-11,95 \%$ en période plus froide (OIS 5.0). Néanmoins, la corrélation entre les deux courbes n'est pas stricte, comme le montre la figure. On peut en déduire que la composition isotopique du carbone dans la calcite dépend également de facteurs autres que ceux commandant celle de l'oxygène.

La précipitation de calcite dans un réseau souterrain est régie par une suite complexe d'équilibres chimiques réversibles, assortis de fractionnements isotopiques [20]. Les différentes réactions et leur cinétique ont été résumées récemment par Emblanch [7] à partir des travaux classiques [20,21,31]. Le modèle, qui fait intervenir les différents réservoirs et qui quantifie le transfert des isotopes du carbone, est notamment développé par Genty et al. [12]. Le rapport isotopique du carbone dans un spéléothème est la résultante du marquage de chaque réservoir carboné intervenant dans la suite réactionnelle et du fractionnement à chaque transition. Les réservoirs de carbone sont le $\mathrm{CO}_{2}$ de l'air, le $\mathrm{CO}_{2}$ du sol et le $\mathrm{CaCO}_{3}$ de l'encaissant carbonaté.

Le $\delta^{13} \mathrm{C}$ de la matrice carbonatée est constant dans un réseau creusé dans un même type de terrain; pour des carbonates marins, il varie entre +2 et $-2 \%$ [7]. Le $\delta^{13} \mathrm{C}$ du $\mathrm{CO}_{2}$ de l'air résulte de l'activité biologique et de l'échange avec l'océan [21]; sa valeur moyenne actuelle est voisine de -7 à $-8 \%$ [6]. Celui du $\mathrm{CO}_{2} \mathrm{du}$ sol est lié à l'importance et à la nature du couvert végétal, qui métabolise plutôt l'isotope léger, et à l'activité bactérienne qui décompose la matière organique.

D'après Lauritzen et Lundberg [16], la composition isotopique du carbone d'un spéléothème est conditionnée par la roche mère, l'activité biologique, le type de cycle photosynthétique, les précipitations et la vitesse de suintement de l'eau alimentant la concrétion. On voit que, pour une cavité donnée, le $\delta^{13} \mathrm{C}$ de la calcite peut donner des indications sur le climat et la végétation 


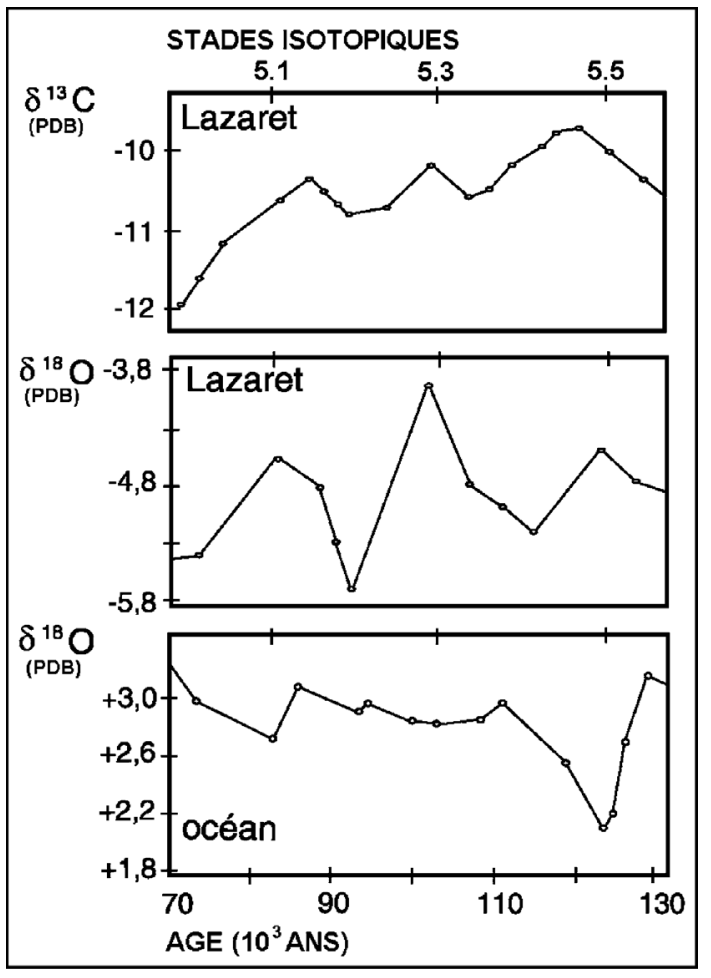

Fig. 4. Variation du $\delta^{13} \mathrm{C}$ et du $\delta^{18} \mathrm{O}$ de la calcite dans l'épaisseur du plancher $\mathrm{E}$, comparée à celle du $\delta^{18} \mathrm{O}$ du test de foraminifères benthiques d'une carotte marine [25].

Fig. 4. $\delta^{13} \mathrm{C}$ and $\delta^{18} \mathrm{O}$ variations in calcite from stalagmitic floor $\mathrm{E}$ compared with marine benthic foraminifer data [25].

contemporaine de la concrétion. Néanmoins, ce signal doit être utilisé avec beaucoup de précaution, car peuvent intervenir le dégazage du $\mathrm{CO}_{2}$ dans l'aquifère et le temps de résidence de l'eau dans le sol, l'âge de la matière organique du sol et l'intensité de la dissolution de la roche encaissante [12].

La nature du cycle photosynthétique chez les plantes a un impact sur la composition isotopique du $\mathrm{CO}_{2}$ qu'elles libèrent au cours de leur décomposition [23, 31]. Les plantes dont le cycle est à trois carbones (plantes en $\mathrm{C} 3$ ) produisent un $\mathrm{CO}_{2}$ très appauvri en ${ }^{13} \mathrm{C}$. Les plantes dont le cycle est à quatre carbones (plantes en $\mathrm{C} 4$ ) produisent un $\mathrm{CO}_{2}$ moins appauvri. Les plantes de type CAM (Crassulean Acid Metabolism) fournissent des valeurs intermédiaires, mais sont trop minoritaires pour influencer le bilan isotopique global. Les valeurs données par Cerling et al. [2] sont une moyenne de $-2,2 \%$ PDB pour les plantes en C3 et $-8,5 \%$ PDB pour les plantes en $\mathrm{C} 4$. Une variation du $\delta^{13} \mathrm{C}$ devrait être enregistrée par la calcite en cas de changement de paléoflore.
Sous les latitudes moyennes, un problème se pose quant à la répartition des plantes en $\mathrm{C} 3$ et $\mathrm{C} 4$. Les plantes en $\mathrm{C} 4$ sont en majorité des monocotylédones et sont nombreuses actuellement dans la prairie et la savane. Sous couvert forestier, les plantes en C3 sont quasi exclusives [3,5]. Dans le carbonate de la matrice du loess en Chine, les variations du $\delta^{13} \mathrm{C}$ sont reliées à la dominance des plantes en $\mathrm{C} 3$ en période interglaciaire (valeur minimale) et des plantes en $\mathrm{C} 4$ en période froide et sèche (valeur maximale) [35]. En région semi-aride, selon Talma et Vogel [32], les valeurs minimales correspondent aux stades glaciaires (forêt en C3) et les valeurs maximales aux interglaciaires chauds et secs (couverture herbeuse en $\mathrm{C} 4$ ).

Il reste à savoir si l'on peut envisager l'alternance des plantes en $\mathrm{C} 3$ et en $\mathrm{C} 4$ dans la région de la grotte du Lazaret. Emblanch [7] considère que le marquage isotopique actuel du $\mathrm{CO}_{2}$ du sol dans le Sud de la France est celui des plantes en $\mathrm{C} 3$ exclusivement. Dans les spectres polliniques [11], la part des plantes en $\mathrm{C} 4$ paraît négligeable devant l'importance des plantes en C3. Les variations enregistrées dans la calcite sont plutôt le reflet des conditions de précipitation et de température influençant la productivité végétale des plantes en C3 et le taux de décomposition de la matière organique du sol. Les valeurs du $\delta^{13} \mathrm{C}$ de la calcite sont à mettre en parallèle avec celles du $\mathrm{CO}_{2}$ des eaux karstiques actuelles, qui varient entre 10 et $14 \%$ environ en région méditerranéenne et dont le $\mathrm{CO}_{2}$ est d'origine biogénique [7]. Dans cette hypothèse, l'impact de la végétation sur le ${ }^{13} \mathrm{C}$ du $\mathrm{CO}_{2}$ du sol serait plus sensible pendant les périodes plus fraîches des sous-stades IOS 5.2 et 5.4, avec des eaux d'infiltration appauvries en ${ }^{13} \mathrm{C}$. On peut supposer que le passage des perturbations atlantiques déterminait un climat tempéré frais et humide, propice à la forêt de feuillus et résineux et aux sols forestiers riches en humus. En revanche, pendant les sous-stades IOS 5.1, 5.3 et 5.5, le climat était plutôt de type méditerranéen, comme aujourd'hui, avec une forêt moins dense et des sols pauvres en humus : le fractionnement isotopique produit par la végétation et retrouvé dans ses produits de décomposition aurait ainsi un impact plus faible sur la composition des eaux d'infiltration. Les fluctuations observées exprimeraient donc les modifications de la couverture végétale au cours du stade 5 .

\subsection{Stérols, terpénoïdes et acides humiques}

Ces molécules organiques peuvent être piégées dans la calcite. Dans le cas du plancher stalagmitique E, elles ont pu être détectées [28]. Les concentrations de ces composés étant très faibles (moins de $1 \mathrm{ng}$ par gramme 
de calcite pour les stérols), les échantillons doivent être de grande taille (quelques centaines de grammes) : ils occupent plusieurs centimètres d'épaisseur dans le plancher et regroupent l'information sur plusieurs milliers d'années. Il ne sera donc pas possible de suivre les variations de ces paramètres au cours du concrétionnement.

L'analyse des stérols montre la présence constante de deux molécules : le cholestérol et le $\beta$-sitostérol. Le cholestérol est d'origine animale; son abondance relative est à rapprocher de la grande quantité d'ossements animaux trouvée dans la grotte. Le $\beta$-sitostérol est caractéristique des végétaux supérieurs; il signifie la présence d'un couvert végétal dans l'environnement proche de la grotte. Le spectre de chromatographie montre également les pics du campéstérol et de produits de dégradation, comme le $\beta$-sitostanol et le stigmastanol. La concentration et le type des stérols dans le site du Lazaret sont sensiblement les mêmes que ceux qui ont été analysés dans un plancher en cours de concrétionnement dans une galerie de carrière à Meudon (banlieue parisienne). Cette analogie suggère (i) que les stérols ont été bien préservés dans la calcite, donc que ce milieu est thermodynamiquement clos, et (ii) que la végétation du Lazaret, pendant l'interglaciaire du stade isotopique 5 , était quantitativement proche de celle existant actuellement en région parisienne.

Les acides humiques, qui constituent la majeure partie du carbone organique des sols, ont été également trouvés dans la calcite, dont ils modifient la couleur [27]. Ils soulignent la présence d'un sol à humus évolué au-dessus de la grotte, comme dans le cas d'un sol forestier.

En outre, l'analyse chromatographique a mis en évidence deux terpénoïdes : l'acide abiétique caractéristique du pin et la bétuline, caractéristique du bouleau. Ces deux taxons sont abondants dans les spectres polliniques [11], mais la mobilité des grains de pollen ne permet pas de conclure à la proximité de ces arbres par rapport à la grotte. Ces deux composés, comme les stérols, n'existent que dans l'appareil végétatif ; leur présence permet donc d'affirmer que des pins et des bouleaux prospéraient dans l'environnement immédiat de la grotte. Leur transport a pu se faire par les eaux d'infiltration, comme pour les acides humiques, mais on ne peut exclure que des bois aient été apportés sur place par les occupants de la grotte.

\section{Conclusions}

Les données isotopiques et biogéochimiques fournies par l'analyse du plancher stalagmitique E apportent des précisions utiles dans la connaissance du paléoenvironnement de la grotte du Lazaret au cours de l'interglaciaire du stade isotopique 5, c'est-à-dire entre -130 et $-70 \mathrm{ka}$ BP environ.

Les variations du $\delta^{18} \mathrm{O}$ apparaissent synchrones de celles enregistrées dans les carottes marines, mais de sens opposé, bien que les comparaisons soient difficiles, $\mathrm{du}$ fait de l'origine variable des sources. Elles illustrent l'alternance des sous-stades chauds et froids du stade OIS 5. Elles seraient la conséquence du changement de circulation océanique et atmosphérique, qui modifierait la source des précipitations. Pendant un sous-stade chaud, les précipitations seraient d'origine méditerranéenne, alors qu'elles seraient d'origine atlantique pendant un sous-stade froid.

Les variations du $\delta^{13} \mathrm{C}$ sont corrélées positivement avec celles du $\delta^{18} \mathrm{O}$. Elles traduiraient les variations du couvert végétal et du type de sol en fonction des fluctuations climatiques. La végétation serait de type arboré avec pins et bouleaux, comme l'indiquent les spectres polliniques et comme le confirment les molécules organiques piégées dans la calcite : stérols, terpénoïdes, acides humiques. Bien que d'interprétation plus délicate, les variations du $\delta^{13} \mathrm{C}$ d'un spéléothème peuvent ainsi refléter les fluctuations des paramètres climatiques, comme l'ont montré Frappier et al. [9] ou Genty et al. [13] pour les événements climatiques millénaires de la dernière période glaciaire.

\section{Références}

[1] M. Bar-Matthews, A. Ayalon, A. Matthews, E. Sass, L. Halicz, Carbon and oxygen isotope study of the active water-carbonate system in a karstic Mediterranean cave: Implications for paleoclimate research in semiarid regions, Geochim. Cosmochim. Acta 60 (2) (1996) 337-347.

[2] T.E. Cerling, J. Quade, Y. Wang, J.R. Bowman, Carbon isotopes in soils and palaeosols as ecology and palaeoecology indicators, Nature 342 (1989) 138-139.

[3] J.A. Dorale, R.L. Edwards, E. Ito, L. Gonzales, Climate and vegetation history of the midcontinent from 75 to $24 \mathrm{ka}$ : A speleothem record from Crevice cave, Missouri, USA, Science 282 (1998) 1871-1874.

[4] J.C. Duplessy, G. Delibrias, J.-L. Turon, C. Pujol, J. Duprat, Deglacial warming of the Northeastern Atlantic ocean: correlation with the paleoclimatic evolution of the European continent, Palaeogeogr. Palaeoclimatol. Palaeoecol. 35 (1981) 121-144.

[5] J. Ehleringer, What controls the abundance of C4 plants?, in: Snowbird Symposium, Utah, États-Unis, décembre 2001, résumé.

[6] J.R. Ehleringer, N. Buchmann, L.B. Flanagan, Carbon isotope ratios in belowground carbon cycle processes. Dept. of Biology, University of Utah, Salt Lake City, États-Unis, 1999, 29 p.

[7] C. Emblanch, Les équilibres chimiques et isotopiques du carbone dans les aquifères karstiques : étude en région méditerranéenne de montagne, thèse, université d'Avignon, 1997, 197 p. 
[8] C. Falguères, H. de Lumley, J.-L. Bischoff, U-series dates on stalagmitic flowstone E (Riss/Würm interglacial) at Grotte du Lazaret, Nice, Quat. Res. 38 (1992) 227-233.

[9] A. Frapier, D. Sahagian, L.A. Gonzales, S.J. Carpenter, El Niño events recorded by stalagmite carbon isotopes, Science 298 (2002) 565 .

[10] A. Frumkin, I. Carmi, A. Gopher, D.C. Ford, H.P. Schwarcz, T. Tsuk, A Holocene millennial-scale climatic cycle from a speleothem in Nahal Qanah Cave, Israel, The Holocene 9 (6) (1999) 677-682.

[11] A. Gauthier, Paléoenvironnements du Pléistocène moyen dans le Sud de la France. Apport et limites de l'analyse pollinique de trois sites préhistoriques : caune de l'Arago, Orgnac 3, grotte du Lazaret, thèse d'État, Institut de paléontologie humaine, Muséum national d'histoire naturelle, 1992, $265 \mathrm{p}$.

[12] D. Genty, A. Baker, M. Massault, C. Proctor, M. Gilmour, E. Pons-Branchu, B. Hamelin, Dead carbon in stalagmites: Carbonates bedrock paleodissolution vs. ageing of soil organic matter. Implications for ${ }^{13} \mathrm{C}$ variations in speleothems, Geochim. Cosmochim. Acta 65 (20) (2001) 3443-3457.

[13] D. Genty, D. Blamart, R. Ouahdi, M. Gilmour, A. Baker, J. Jouzel, S. Van-Exter, Precise dating of Dansgaard-Oeschger climate oscillations in Western Europe from stalagmite data, Nature 421 (2003) 833

[14] K.R. Johnson, B.L. Ingram, High-resolution paleoclimate record based on $\delta^{18} \mathrm{O}$ variations in speleothems from central China, Dept. Earth Planet. Sci., Univ. Berkeley, CA, 2002, 3 p.

[15] J. Labeyrie, Le cadre paléoclimatique depuis 140000 ans, Rev. Anthropol. 88 (1984) 19-48.

[16] S.E. Lauritzen, J. Lundberg, Speleothems and climate: a special issue of the Holocene, The Holocene 9 (6) (1999) 643-647.

[17] H. de Lumley, La place du remplissage de la grotte du Lazaret (A-M) dans la stratigraphie du Quaternaire de la région de Nice à Monaco, Bull. Mus. Anthropol. Préhist. Monaco 8 (1961) 97133.

[18] H. de Lumley, A. Tavoso, La stratigraphie des couches supérieures de la grotte du Lazaret, Mém. Soc. Préhist. Fr. (1969) $17-24$.

[19] M.-A. Lumley-Woodyear, Anténéandertaliens et néandertaliens du Bassin méditerranéen occidental européen, thèse d'État, faculté des sciences de Paris, 1970, pp. 80-148.

[20] W.G. Mook, J.C. Bommerson, W.H. Staverman, Carbon isotopes fractionation between dissolved bicarbonate and gaseous carbon dioxide, Earth Planet. Sci. Lett. 22 (1974) 169-176.

[21] W.G. Mook, M. Koopmans, A.F. Carter, C.D. Keeling, Seasonal, latitudinal, and secular variations in the abundance and isotopic ratios of atmospheric carbon dioxide. Results from land stations, J. Geophys. Res. 88 (1983) 10915-10933.

[22] F.C.E. Octobon, Grotte du Lazaret (A-M). Huitième étude sur les fouilles exécutées dans le locus VIII de cette grotte (ancienne grotte Lympia) et sur la géologie quaternaire locale, Bull. Mus. Anthropol. Préhist. Monaco 12 (1955) 23-101.

[23] M.H. O'Leary, Carbon isotope fractionation in plants, Phytochemistry 20 (4) (1981) 553-567.

[24] J.R. O'Neil, R.N. Clayton, T.K. Mayeda, Oxygen isotopes fractionation in divalent metal carbonates, J. Chem. Phys. 51 (1969) 5547-5558.

[25] N.G. Pisias, D.G. Martinson, T.C. Moore, N.J. Shackleton, W. Prell, J. Hays, G. Boden, High-resolution stratigraphic correlation of benthic oxygen isotopic records spanning the last 300000 years, Mar. Geol. 56 (1984) 119-156.

[26] J. Quin, Studies on oxygen isotope thermometry of cave sediment and paleoclimatic record, Network Center Guangxi Normal University, 1999, 9 p.

[27] L. Rousseau, O. Bouloussa, H. de Lumley, Évaluation de l'influence respective des acides humiques et des oxydes ferriques sur la coloration des planchers stalagmitiques, C. R. Acad. Sci. Paris, Ser. II 317 (1993) 367-370.

[28] L. Rousseau, S. Laafar, C. Pepe, H. de Lumley, Sterols as biogeochemical markers: results from 'Ensemble E' of the stalagmitic floor, grotte du Lazaret, Nice, France, Quat. Sci. Rev. 14 (1995) $51-59$.

[29] N.J. Shackleton, Attainment of isotopic equilibrium between ocean water and the benthonic foraminifera genus Uviregina: Isotopic change in the ocean during the last glacial, in: J. Labeyrie (Ed.), Les méthodes quantitatives d'études des variations du climat au cours du Pléistocène, coll. int., CNRS, Paris, 1974, pp. 203-209, 219.

[30] G. Shen, Datation des planchers stalagmitiques des sites acheuléens en Europe par les méthodes des déséquilibres des familles de l'uranium et contribution méthodologique, thèse d'État, Institut de paléontologie humaine, université Pierre-et-Marie-Curie, Paris-6, 1985, 173 p.

[31] W. Solomon, W.G. Mook, Isotope geochemistry of carbonates in the weathering zone, in: P. Fritz, J.-C. Fontes (Eds.), Handbook of Environmental Isotope Geochemistry, vol. 2-B, 1986, pp. 239-269.

[32] A.S. Talma, J.C. Vogel, Late Quaternary paleotemperatures derived from a speleothem from Cango Caves, Cape Province, South Africa, Quat. Res. 37 (1992) 203-213.

[33] P.W. Williams, A. Marshall, D.C. Ford, A.V. Jenkinson, Paleoclimatic interpretation of stable isotope data from Holocene speleothems of the Waitomo district, North Island, New Zealand, The Holocene 9 (6) (1999) 649-657.

[34] I.J. Winograd, T.B. Coplen, J.M. Landwehr, A.C. Riggs, K.R. Ludwig, B.J. Szabo, P.T. Kolesar, K.M. Revesz, Continuous 500,000 -year climate record from vein calcite in Devil's Hole, Nevada, Science 258 (1992) 255-260.

[35] L. Zheng-Hua, C.I. Moral, W. Sue, History of atmospheric $\mathrm{CO}_{2}$ and the impact on plants, animals and ecosystems, in: Snowbird Symposium, Utah, décembre 2001, résumé. 